\title{
Beam propagation method and its application to integrated optic structures and optical fibers
}

\author{
SHIVA KUMAR, T SRINIVAS and A SELVARAJAN \\ Department of Electrical Communication Engineering, Indian Institute of Science, Bangalore \\ 560012 , India
}

MS received 28 July 1989; revised 10 November 1989

\begin{abstract}
Practical applications of integrated optics require understanding of light propagation in dielectric waveguides of various geometries and calls for elegant and quick methods of analysis. In this paper, we use beam propagation method to analyse some integrated optic waveguiding elements such as waveguide with bend and branching waveguide. The method is extended to cylindrical co-ordinates, so that structures with circular symmetry can be easily solved. We first present a general beam propagation method algorithm, followed by results taking typical values for various parameters. Our studies show that the efficiency of the method depends on the $z$-propagation steps and on the number of points chosen for the Fourier transform. The algorithm developed can be used to analyse many other integrated optic structures and to study the effect of other input beam profiles.
\end{abstract}

Keywords. Integrated optics; branching waveguide; Hankel transform; fast fourier transform.

PACS No. $\quad \mathbf{4 2 \cdot 8 2}$

\section{Introduction}

Beam propagation method is basically a numerical modelling method for the propagation of an optical beam through a medium with small variations of refractive index (Feit and Fleck 1978; Van Roey et al 1981). The method consists of propagating the input beam over a small distance through a homogeneous space and then correcting for the refractive index variations seen by this beam during the propagation step. In this paper we give an algorithm for generalized beam propagation method (BPM) and apply it to some integrated optic structures and optical fibers. To solve the wave equation of the homogeneous medium, the Fourier transform pair has to be evaluated. Instead of using fast Fourier transform (FFT) directly, it is suggested that FFT combined with Simpson's $1 / 3$ rule can be used which improves the accuracy. Also, for optical fibers the Hankel transform method can be used instead of the two-dimensional FFT.

\section{Theory}

The propagation of light in a waveguide can be described by the wave equation

$$
\frac{\partial^{2} \psi}{\partial x^{2}}+\frac{\partial^{2} \psi}{\partial z^{2}}+k_{0}^{2} n^{2}(\omega, x) \psi=0
$$


where $k_{0}=\omega / c, n$ the refractive index and the $\omega$ circular frequency of light. The solution to equation (1) at $z=\Delta z$ may be written in terms of field at $z=0$ as,

$$
\psi(x, \Delta z)=\exp \left[-i \Delta z\left\{\nabla_{T}^{2}+k_{0}^{2} n^{2}\right\}^{1 / 2}\right] \psi(x, 0),
$$

where $\nabla_{T}^{2}=\partial^{2} / \partial x^{2}$. The solution $\psi$ can be expressed in the form,

$$
\psi(x, z)=\xi(x, z) \exp (-i k z)
$$

where $k=n_{0} \omega / c$ and $n_{0}$ is the refractive index of the homogeneous medium. $\xi$ can be written in the symmetrized split operator form to second order $\Delta z$ (Feit and Fleck 1976) as

$$
\begin{aligned}
\xi(x, \Delta z)= & \exp \left\{-\frac{i \Delta z}{2}\left[\frac{\nabla_{T}^{2}}{\left(\nabla_{T}^{2}+k^{2}\right)^{1 / 2}+k}\right]\right\} \exp (-i \Delta z \chi) \\
& \times \exp \left\{\frac{-i \Delta z}{2}\left[\frac{\nabla_{T}^{2}}{\left(\nabla_{T}^{2}+k^{2}\right)^{1 / 2}+k}\right]\right\} \xi(x, 0)+0(\Delta z)^{3},
\end{aligned}
$$

where $\chi=k_{0} *\left[n(x)-n_{0}\right]$. The operation

$$
\exp \left\{-i \Delta z\left[\frac{\nabla_{T}^{2}}{\left(\nabla_{T}^{2}+k^{2}\right)^{1 / 2}+k}\right]\right\} \xi(x, 0)
$$

is equivalent to solving the wave equation

$$
\frac{\partial^{2} \psi}{\partial x^{2}}+\frac{\partial^{2} \psi}{\partial z^{2}}+k_{0}^{2} n_{0}^{2} \psi=0
$$

with $\psi(x, 0)$ as an initial condition. Van Roey et al (1981) and Van der Donk (1982) have however used a simple correction factor

$$
\psi\left(x, z_{0}+\Delta z\right)=\eta\left(x, z_{0}+\Delta z\right) \exp \left(-i k_{0} * \Delta n(x) * \Delta z\right)
$$

where $\Delta n(x)=n(x)-n_{0}$ and $\eta(x, z)$ satisfies,

$$
\frac{\partial^{2} \eta}{\partial x^{2}}+\frac{\partial^{2} \eta}{\partial z^{2}}+k_{0}^{2} n_{0}^{2} \eta=0
$$

and $\eta\left(x, z_{0}\right)=\psi\left(x, z_{0}\right)$. Equation (7) can be solved by Fourier transform pairs (Lee 1986)

$$
\begin{aligned}
& \eta\left(x, z_{0}+\Delta z\right)=\frac{1}{2 \pi} \int_{-\infty}^{\infty} \bar{\eta}\left(k_{x}, z_{0}+\Delta z\right) \exp \left(j k_{x} x\right) \mathrm{d} k_{x}, \\
& \bar{\eta}\left(k_{x}, z_{0}\right)=\int_{-\infty}^{\infty} \eta\left(x, z_{0}\right) \exp \left(-j k_{x} x\right) \mathrm{d} x, \\
& \bar{\eta}\left(k_{x}, z_{0}+\Delta z\right)=\bar{\eta}\left(k_{x}, z_{0}\right) \exp (-j \beta \Delta z),
\end{aligned}
$$

where $\beta=\left(k_{0}^{2} n_{0}^{2}-k_{x}^{2}\right)^{1 / 2}$. Equation (8) is substituted in (6) to get the desired field $\psi$ at 
$z_{0}+\Delta z$. It is known that the error introduced while using (4) is $\sim \Delta z^{3}$ and while using (6) is $\sim \Delta z^{2}$. Equation (8) has to be solved in both the cases. To solve (8) and (9), Simpson's $1 / 3$ rule can be used. But it would need $2 N+1$ complex multiplications and additions for each value of $k_{x}$ where $2 N$ is the number of samples taken for integration. If $\bar{n}\left(k_{x}\right)$ is calculated $2 N$ times, then $2 N(2 N+1)$ complex multiplication and addition will be needed. To reduce the number of complex multiplications, Simpson's $1 / 3$ rule is combined with FFT so that the total number of complex multiplications reduces to $4 N \log N$ (Appendix A). Thus we get the accuracy of Simpson's $1 / 3$ rule and the speed of FFT at the same time.

\section{Extracting mode data}

The complex field amplitude can be expressed as a superposition of orthogonal mode eigen functions,

$$
\psi(x, z)=\sum_{n, j} A_{n j} u_{n j}(x) \exp \left(-i \beta_{n} z\right)
$$

where $n$ is a mode index, $j$ the distinguishing members of degenerate group and $A_{n j}$ is determined by the input field $\psi(x, 0)$; The mode propagation constant $\beta_{n}$ can be determined from computation of correlation function, (Feit and Fleck 1978)

$$
P_{1}(z)=\int_{-\infty}^{\infty} \psi^{*}(x, 0) \psi(x, z) \mathrm{d} x .
$$

Using (11) and the orthogonality of mode eigen functions, (12) reduces to,

$$
P_{1}(z)=\sum_{n, j}\left|A_{n j}\right|^{2} \exp \left(-i \beta_{n} z\right)
$$

Multiplying (13) by the Hanning window function,

$$
\begin{aligned}
w(z) & =1-\cos \left(2 \cdot 0 * \pi * z / Z_{1}\right) & & 0<=z<=Z_{1} \\
& =0 & & \text { otherwise }
\end{aligned}
$$

and taking Fourier transform w.r.t. $z$,

$$
\begin{aligned}
\bar{P}_{1}(\beta) & =\frac{1}{Z_{1}} \int_{0}^{z_{1}} P_{1}(z) w(z) \exp (-i \beta z) \mathrm{d} z \\
= & \sum_{n, j}\left|A_{n j}\right|^{2} \mathscr{L}\left(\beta-\beta_{n}\right), \\
\mathscr{L}\left(\beta-\beta_{n}\right)= & \frac{\exp \left[i\left(\beta-\beta_{n}\right) z_{1}\right]-1}{i\left(\beta-\beta_{n}\right) z_{1}} \\
& -\frac{1}{2}\left\{\frac{\exp \left[i\left(\beta-\beta_{n}\right) z_{1}+2 \pi\right]-1}{i\left(\beta-\beta_{n}\right) z_{1}+2 \pi}+\frac{\exp \left[i\left(\beta-\beta_{n}\right) z_{1}-2 \pi\right]-1}{i\left(\beta-\beta_{n}\right) z_{1}-2 \pi}\right\} .
\end{aligned}
$$

The eigenvalue $\beta_{n}$ can be determined by locating the local maxima of $\bar{P}_{1}(\beta)$. 


\section{Extensions of BPM to cylindrical coordinates}

Consider the wave equation in cylindrical co-ordinates.

$$
\frac{\partial^{2} \psi}{\partial r^{2}}+\frac{1}{r} \frac{\partial \psi}{\partial r}+\frac{1}{r^{2}} \frac{\partial^{2} \psi}{\partial \theta^{2}}+\frac{\partial^{2} \psi}{\partial z^{2}}+k_{0}^{2} n^{2}(r) \psi=0
$$

which can be written as (Feit and Fleck 1976)

$$
\psi(r, \theta, z)=\exp \left(-i\left(\nabla_{T}^{2}+k_{0}^{2} n^{2}\right)^{1 / 2} z\right) \psi(r, \theta, 0)
$$

where $\nabla_{T}^{2}=\partial^{2} / \partial r^{2}+1 / r \partial / \partial r+\left(1 / r^{2}\right) \partial^{2} / \partial \theta^{2}\left(\nabla_{T}^{2}+k_{0}^{2} n^{2}\right)^{1 / 2}$ can be written in the form,

$$
\frac{\left(\nabla_{T}^{2}+k_{0}^{2} n^{2}\right)^{1 / 2}\left[\left(\nabla_{T}^{2}+k_{0}^{2} n^{2}\right)^{1 / 2}+k_{0} n\right]}{\left[\left(\nabla_{T}^{2}+k_{0}^{2} n^{2}\right)^{1 / 2}+k_{0} n\right]}=\frac{\nabla_{T}^{2}}{\left[\left(\nabla_{T}^{2}+k_{0}^{2} n^{2}\right)^{1 / 2}+k_{0} n\right]}+k_{0} n
$$

If $n$ in the first term of the right hand side is approximated by $n_{0}$, where $n_{0}$ is the refractive index of the unperturbed medium, equation (18) becomes

$$
\begin{aligned}
\left(\nabla_{T}^{2}+k_{0}^{2} n^{2}\right)^{1 / 2} & \cong\left[\frac{\nabla_{T}^{2}}{\left(\nabla_{T}^{2}+k_{0}^{2} n_{0}^{2}\right)^{1 / 2}+k_{0} n_{0}}+k_{0} n_{0}\right]+k_{0}\left[n(r)-n_{0}\right] \\
& \cong\left[\nabla_{T}^{2}+k_{0}^{2} n_{0}^{2}\right]^{1 / 2}+k_{0} \Delta n(r) \text { from }(18)
\end{aligned}
$$

where $\Delta n(r)=n(r)-n_{0}$. Substituting in (17),

$$
\psi(r, \theta, z)=\exp \left[\left\{-\mathrm{i} z\left(\nabla_{T}^{2}+k_{0}^{2} n_{0}^{2}\right)^{1 / 2}\right\}-\left\{i z * k_{0} * \Delta n(r)\right\}\right] \psi(r, \theta, 0) .
$$

By neglecting the error due to non-commutation of operators, (19) can be written as,

$$
\begin{aligned}
\psi(r, \theta, z) & \cong\left[\exp \left[-i z\left(\nabla_{T}^{2}+k_{0}^{2} n_{0}^{2}\right)^{1 / 2}\right] \psi(r, \theta, 0)\right] \exp \left[-i z * k_{0} * \Delta n(r)\right] \\
& \cong \phi(r, \theta, z) * \exp \left[-i z * k_{0} \Delta n(r)\right],
\end{aligned}
$$

where $\phi$ satisfies the wave equation

$$
\frac{\partial^{2} \phi}{\partial r^{2}}+\frac{1}{r} \frac{\partial \phi}{\partial r}+\frac{1}{r^{2}} \frac{\partial^{2} \phi}{\partial \theta^{2}}+\frac{\partial^{2} \phi}{\partial z^{2}}+k_{0}^{2} n_{0}^{2} \phi=0
$$

with $\phi(r, \theta, 0)$ as initial condition. Let

$$
\phi=R(r, z) *[A \cos m \theta+B \sin m \theta] .
$$

Then, (21) becomes,

$$
\frac{\partial^{2} R}{\partial r^{2}}+\frac{1}{r} \frac{\partial R}{\partial r}+\frac{\partial^{2} R}{\partial z^{2}}+\left[k_{0}^{2} n_{0}^{2}-\frac{m^{2}}{r^{2}}\right] R=0 .
$$

Taking the Hankel transform,

$$
\frac{\mathrm{d}^{2} \bar{R}(\alpha, z)}{\mathrm{d} z^{2}}+\left(k_{0}^{2} n_{0}^{2}-\alpha^{2}\right) \bar{R}(\alpha, z)=0
$$


where

$$
\begin{aligned}
& \bar{R}(\alpha, z)=\int_{0}^{\infty} R(r, z) r J_{m}(\alpha r) \mathrm{d} r \\
& \bar{R}(\alpha, z)=\bar{R}(\alpha, 0) \exp (-j \beta z) \text { where } \beta=\left(k_{0}^{2} n_{0}^{2}-\alpha^{2}\right)^{1 / 2}
\end{aligned}
$$

Taking the inverse Hankel transform,

$$
R(r, z)=\int_{0}^{\infty}[\bar{R}(\alpha, 0) \exp (-j \beta z)] \alpha J_{m}(\alpha r) \mathrm{d} \alpha .
$$

For structures such as weakly guiding single-mode fibers, we can set $m=0$.

$$
\begin{aligned}
& R(r, z)=\int_{0}^{\infty}[\bar{R}(\alpha, 0) \exp (-j \beta z)] \alpha J_{0}(\alpha r) \mathrm{d} \alpha \\
& \bar{R}(\alpha, 0)=\int_{0}^{\infty} R(r, z) r J_{0}(\alpha r) \mathrm{d} r .
\end{aligned}
$$

To evaluate (26) and (27), the Fast Hankel transform technique is used (Appendix B). Hence $\phi$ is calculated and substituted in (20) to get the desired field.

\section{Results}

To verify the above equations, a step index fiber is considered. The fiber is excited with step input, i.e. the field within the fiber is uniform and is zero outside. The fiber has the following parameters: core refractive index of 1.45 , cladding refractive index of 1.44 , a radius of $2 \mu \mathrm{m}$ and a wavelength of $1 \cdot 32 \mu \mathrm{m}$. To find the eigenvalues, instead of $(12)$

$$
P_{1}(z)=\int_{0}^{\infty} R^{*}(r, 0) R(r, z) r \mathrm{~d} r
$$

is used. It is found to excite only single-guided mode $\beta_{1}=6.868 \mathrm{rad} / \mu \mathrm{m}$. The propagation constant obtained after solving the characteristic equation for weakly guiding fibers is, $\beta_{1}=6.8676 \mathrm{rad} / \mu \mathrm{m}$.

To find the field in the integrated optic structures, we have used the simple correction factor of (6) instead of (4) since the propagation distance is $1000 \mu \mathrm{m}$.

From figure 1(a), it can be seen that the given power input is carried through bent guiding region. The bent waveguide is excited as shown. Power output is calculated for different angles. Power in the bent waveguide for different angles of bend is shown in figure $1(\mathrm{~b})$. For angles greater than $10^{\circ}$, most of the power is radiated out of the waveguide.

Figure 2 shows the field in a branching waveguide. Yajima (1973) experimentally showed that modes incident on an asymmetric planar-dielectric branching waveguide with a shallow taper propagate such that the mode power is transferred to one arm of the branch or the other. The branching waveguide acts like a power divider (non-adiabatic) when tapers are steep and it acts like a mode-splitter (adiabatic) when 


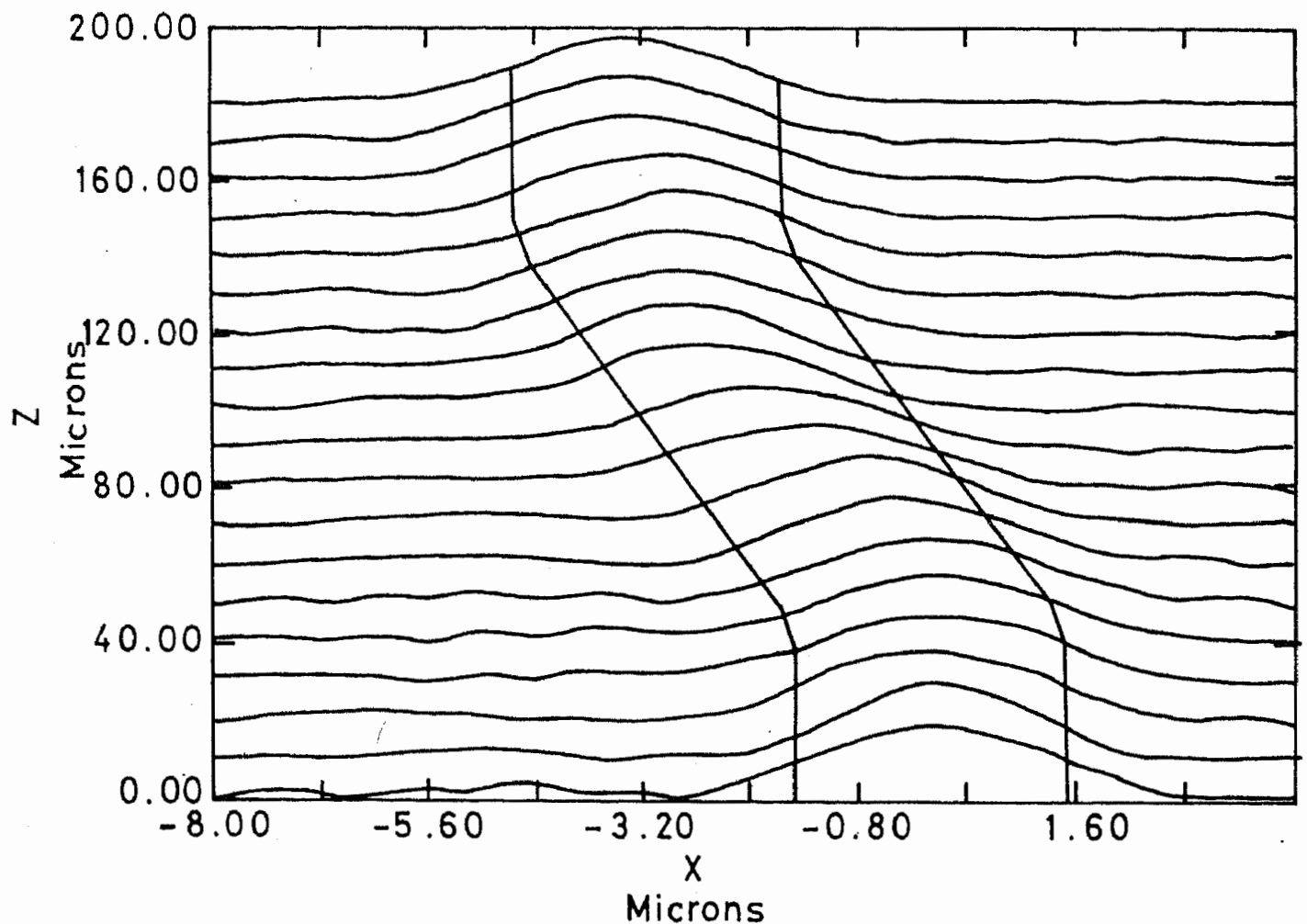

Fig. 1a.

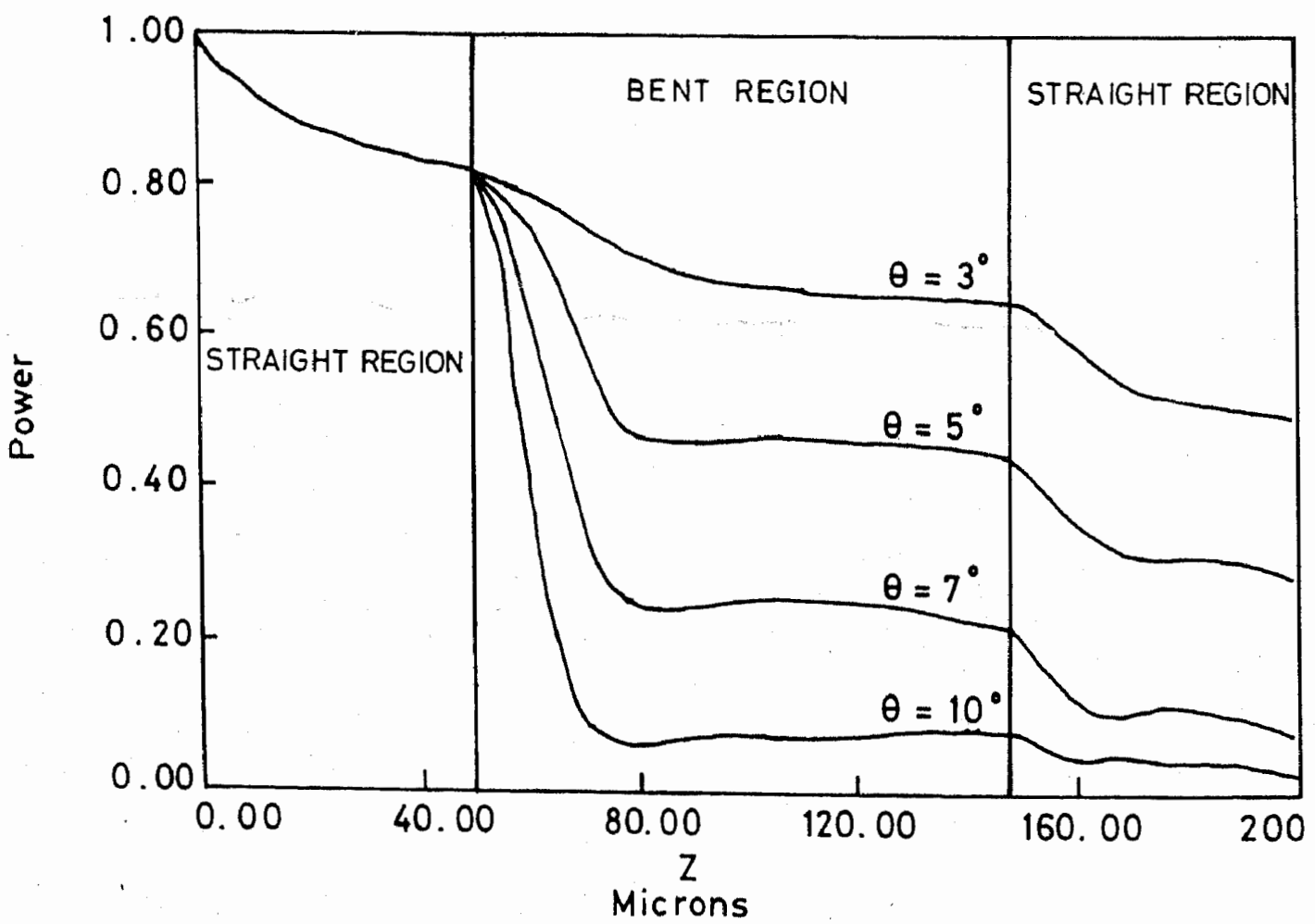

Fig. 1 .

Figure 1a. Field in a bent waveguide for Gaussian input with $1 / e$ depth $2 \mu \mathrm{m} . n_{s}=2 \cdot 1398$. $\Delta n=0.1 \%$. b. Power in a bent waveguide for different angles of bend. 

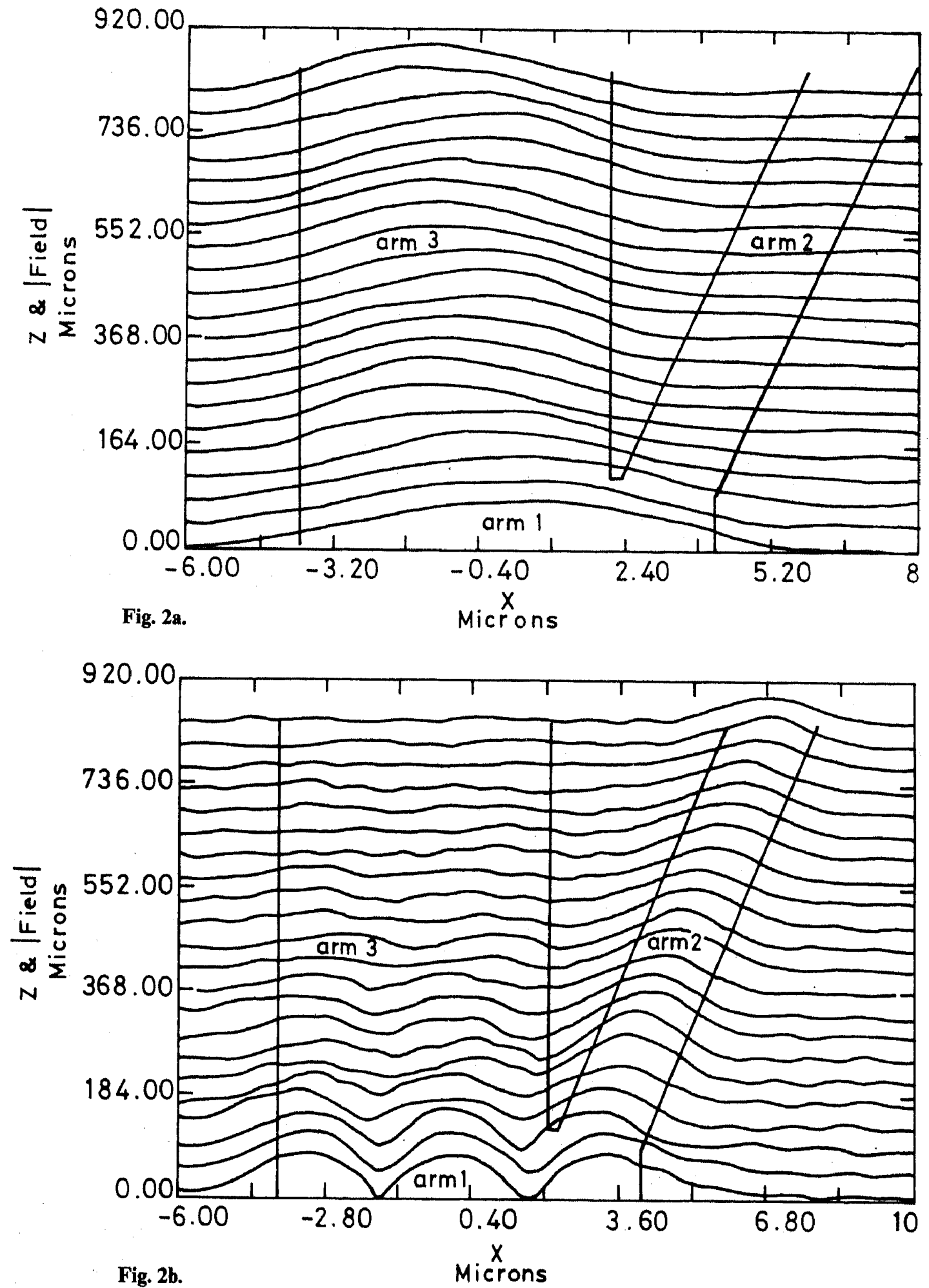

Fig. 2b.

Microns

Figure 2a. Field in a branching waveguide with the fundamental mode excitation into arm 1. The angle is $1 / 200$ radians. The guide thickness for arm 1 and arm 2 is $8 \mu \mathrm{m}$ and $2 \mu \mathrm{m}$ respectively. For arm 1 and arm 3, the refractive indices (RI) within the guide are $2 \cdot 155$ and 2.1398 outside. For arm 2, RI is 2.155 within the guide on the right side and 2.1398 on the left side.

Figure 2b. Field in a branching waveguide with the TE03 mode excitation into arm 1 . The parameters are same as figure $2 \mathrm{a}$. 


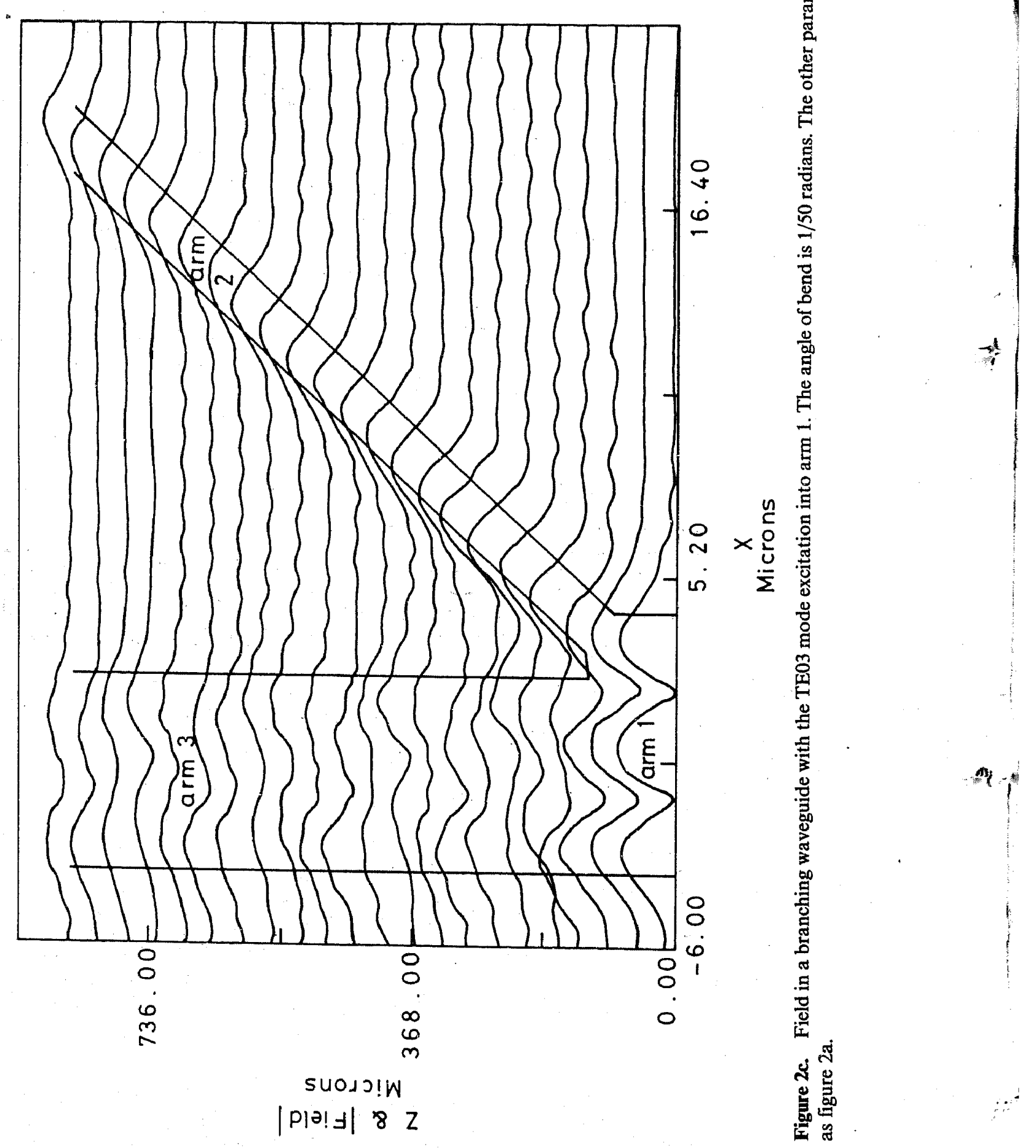


tapers are gradual. Figure 2(a) shows the field in a branching waveguide for shallow taper. The angle of bend is $1 / 200$ radians. The fundamental mode is launched into arm 1 of the waveguide. The thickness of arm 2 is made much less than that of arm 3 so that propagation constant for fundamental mode in arm 2 is less than that of arm 3. For $\lambda=1.32 \mu \mathrm{m}, \beta=10.2190 \mathrm{rad} / \mu \mathrm{m}$ for arm 2 and $\beta=10.2494 \mathrm{rad} / \mu \mathrm{m}$ for arm 3 for the fundamental mode. In this case, TE01 mode in arm 1 is converted to the TE01 mode in arm 3. Hence, it can be seen that TE01 mode in arm 1 chooses the arm of larger propagation constant for TE01 mode. We can see from 2(a) that power launched in arm 1 is coming out of arm 3.

Figure 2(b) shows the field in the branching waveguide with TE03 mode excitation. The propagation constant for arm 1 is,

$$
\begin{aligned}
& \beta_{11}=10.2524 \mathrm{rad} / \mu \mathrm{m} \text { for TE01 mode, } \\
& \beta_{12}=10.2372 \mathrm{rad} / \mu \mathrm{m} \text { for TE02 mode, } \\
& \beta_{13}=10.2132 \mathrm{rad} / \mu \mathrm{m} \text { for TE03 mode. }
\end{aligned}
$$

For arm 3,

$$
\begin{aligned}
& \beta_{31}=10 \cdot 2494 \mathrm{rad} / \mu \mathrm{m} \text { for TE01 mode, } \\
& \beta_{32}=10 \cdot 2258 \mathrm{rad} / \mu \mathrm{m} \text { for TE02 mode, } \\
& \beta_{33}=10.1924 \mathrm{rad} / \mu \mathrm{m} \text { for TE03 mode. }
\end{aligned}
$$

For arm 2,

$$
\beta_{21}=10 \cdot 2190 \mathrm{rad} / \mu \mathrm{m} \text { for TE01 mode. }
$$

It can be seen that TE03 mode of arm 1 is converted into TE01 mode of arm 2 since the propagation constant for TE01 mode of arm 2 is closest to that for TE03 mode of arm 1.

Figure 2(c) shows the field in the branching waveguide when the tapers are steep. The angle of bend is $1 / 50$ radians. Now, the waveguide acts like a power splitter. After doing the modal analysis, it is found that, in arm 3 , the odd mode with $\beta=10.2257 \mathrm{rad} / \mu \mathrm{m}$ is excited. In arm 2 , the even mode with $\beta=10.219 \mathrm{rad} / \mu \mathrm{m}$ is excited. The ratio of power in arm 2 to arm 3 is found to be $36: 64$.

\section{Conclusions}

Beam propagation method is a powerful tool for the numerical modelling of scalar wave propagation through media with arbitrary but slow and small variations in refractive index. For structures with circular symmetry, the Hankel transform method can be used which reduces the computational time against the two-dimensional Fourier transform.

\section{Appendix A}

Multiply equation (7) by $\exp (-j * 2 \pi k x / T)$ and integrate w.r.t. $x$ with limits $-T / 2$ to 
$T / 2$ where $T$ is a large number such that

where

$$
\begin{aligned}
& \eta( \pm T / 2) \cong \frac{\partial \eta( \pm T / 2)}{\partial x} \simeq 0 \\
& \frac{\partial^{2} \bar{\eta}(k, z)}{\partial z^{2}}+\left[k_{0}^{2} n_{0}^{2}-\left(\frac{2 \pi k}{T}\right)^{2}\right] \bar{\eta}(k, z)=0
\end{aligned}
$$

$$
\begin{aligned}
& \bar{\eta}(k, z)=\frac{1}{T} \int_{-T / 2}^{T / 2} \eta(x, z) \exp (-i 2 \pi k x / T) \mathrm{d} x \\
& \bar{\eta}(k, \Delta z)=\bar{\eta}(k, 0) \exp \left(-j \beta_{k} \Delta z\right) \text { where } \beta_{k}=\left[\left(k_{0}^{2} n_{0}^{2}\right)-(2 \pi k / T)^{2}\right]^{1 / 2} \\
& \bar{\eta}(x, \Delta z)=\sum_{k=-L}^{L} \bar{\eta}(k, 0) \exp \left(-j \beta_{k} \Delta z\right) \exp (i 2 \pi k x / T)
\end{aligned}
$$

where $2 L$ is the total number of prominent Fourier spectral components. To evaluate (A2), Simpson's $1 / 3$ rule is used. Let $h$ be the interval between samples and $2 N$ be the total number of samples. Then (A2) can be written as,

$$
\bar{\eta}(k, 0)=\frac{1}{6 N}\left\{w_{0}+4 \cdot 0\left(w_{1}+w_{3} \cdots+w_{2 N-1}\right)+2 \cdot 0\left(w_{2}+w_{4}+\cdots w_{2 N-2}+w_{2 N}\right\}\right.
$$

where $w(x)=\eta(x, 0) \exp (-j * 2 \pi k * x / T)$. Replacing the continuous variable $x$ with a discrete variable $x=(T * n / 2 N)-T / 2$ (A5) can be written as,

$$
\begin{aligned}
\vec{\eta}(k, 0)= & \frac{\exp (j \pi k)}{6 N}\left[40 \sum_{\substack{n \\
\text { odd }}} \eta_{n} \exp \left(\frac{-i 2 \pi k n}{2 N}\right)\right. \\
& \left.+2 \cdot 0 \sum_{\substack{n \\
\text { even, } \neq 0,2 N}} \eta_{n} \exp \left(\frac{-i 2 \pi k n}{2 N}\right)+\eta_{0}+\eta_{2 N}\right] .
\end{aligned}
$$

Putting $m=(n-1) / 2$ for the odd terms and $m=(n-2) / 2$ for the even terms, we get

$$
\begin{aligned}
\bar{\eta}(k, 0)= & \frac{\exp (j \pi * k)}{6 N}\left[4 \cdot 0 \sum_{m=0}^{N-1} \eta_{2 m+1} \exp \left(\frac{-j 2 \pi k m}{N}\right) \exp (-j \pi k)\right. \\
& \left.+2 \cdot 0 \sum_{m=0}^{N-1} \eta_{2 m+2} \exp \left(\frac{-i 2 \pi k m}{N}\right)+\eta_{0}-\eta_{2 N}\right] \\
= & \frac{1}{6 N}\left[4 \cdot 0 \sum_{m=0}^{N-1} \eta_{2 m+1} \exp \left(\frac{-i 2 \pi k m}{N}\right)\right. \\
& \left.+2 \cdot 0 *(-1)^{k} \sum_{m=0}^{N-1} n_{2 m+2} \exp \left(\frac{-i 2 \pi k m}{N}\right)+\eta_{0}-\eta_{2 N}\right]
\end{aligned}
$$

Using FFT algorithms (Oppenheim and Schafer 1975) for summations in (A7), $\bar{\eta}$ can be evaluated. Each FFT takes $2 N \log N$ complex multiplications. Therefore, the total complex multiplication will be $4 N \log N$. 


\section{Appendix B}

The fast Hankel transform (Siegman 1977) reduces the effort required to calculate Bessel functions for every iteration. $r$ and $\alpha$ of (27) can be replaced by,

and

$$
r=r_{0} \exp (p x), \quad \alpha=\alpha_{0} \exp (p y),
$$

$$
r R(r) \equiv f(x) ; \quad \alpha \bar{R}(\alpha) \equiv g(y),
$$

The Hankel transform,

i.e.

$$
\alpha \bar{R}(\alpha)=\int_{0}^{\infty} J_{0}(\alpha r) \alpha r R(r) \mathrm{d} r
$$

where

$$
\alpha \bar{R}(\alpha) \equiv g(y)=\int_{-\infty}^{\infty} j(x+y) f(x) \mathrm{d} x
$$

$$
j(r)=J_{0}\left(\alpha_{0} r_{0} \exp (p r)\right) p r_{0} \alpha_{0} \exp (p r) .
$$

This change of variable may be called Gardner Transform (Gardner et al 1959). Taking the Fourier transform of (B4),

$$
\begin{aligned}
G(k)= & \int_{-\infty}^{\infty} g(y) \exp (-i k y) \mathrm{d} y=\int_{-\infty}^{\infty} j(x+y) \exp [-i k(x+y)] \\
& \times \int_{-\infty}^{\infty} f(x) \exp (i k y) \mathrm{d} x \mathrm{~d} y \\
= & J(k) * F(-k) .
\end{aligned}
$$

where $J(k)$ and $F(k)$ are the Fourier transforms of $j$ and $f$ respectively. Finally, $g(y)$ can be found by taking the inverse Fourier transform of $G(k)$.

$$
g(y)=\frac{1}{2 \pi} \int_{-\infty}^{\infty} J(k) F(-k) \exp (i k y) \mathrm{d} k .
$$

Thus, the Hankel transform of $R(r)$ requires an FFT on $f(x)$, FFT on $j(x)$, and an inverse FFT to get $g(y)$ or $\bar{R}(\alpha)$. The Fourier transform of the Bessel function needs to be obtained only once. Hence, the calculation of Bessel function for every iteration is avoided.

\section{Acknowledgements}

The authors wish to thank the referee for valuable suggestions to improve the paper.

\section{References}

Feit M D and Fleck Jr J A 1978 Appl. Opt. 173990

Feit M D and Fleck Jr J A 1979 Appl. Opt. 182843 
Gardner D G, Gardner J C, Lausch G and Meinke W W 1959 J. Chem. Phys. 31987

Lee D L 1986 Electromagnetic principles of integrated optics (New York: John Wiley) ch. 2

Oppenheim A V and Schafer R W 1975 Digital signal processing (New Delhi: Prentice-Hall of India) ch. 6 Siegman A E 1977 Opt. Lett. 113

Van der Donk J 1982 The beam propagation method in integrated optics Ph.D. Thesis, University of Gent,

Gent, Belgium

Van Roey J, Van der Donk J and Lagasse P E 1981 J. Opt. Soc. Am. 71803

Yajima H 1973 Appl. Phys. Lett. 22647 